\title{
Comparison of Spinal Cord Stimulation Profiles from Intra- and Extradural Electrode Arrangements by Finite Element Modelling
}

\author{
Qiujun Huang · Hiroyuki Oya · Oliver E. Flouty · Chandan G. Reddy · \\ Matthew A. Howard III · George T. Gillies · Marcel Utz
}

the date of receipt and acceptance should be inserted later

\begin{abstract}
Spinal cord stimulation (SCS) currently relies on extradural electrode arrays that are separated from the spinal cord surface by a highly conducting layer of cerebrospinal fluid. It has recently been suggested that intradural placement of the electrodes in direct contact with the pial surface could greatly enhance the specificity and efficiency of stimulation. The present computational study aims at quantifying and comparing the electrical current distributions as well as the spatial recruitment profiles resulting from extraand intra-dural electrode arrangements. The electrical potential distribution is calculated using a 3D finite element model of the human thoracic spinal canal. The likely recruitment areas are then obtained by using the potential as input to an equivalent circuit model of the pre-threshold axonal response. The results show that the current threshold to recruitment of axons in the dorsal column is more than an order of magnitude smaller for intradural than extradural stimulation. Intradural placement of the electrodes also leads to much higher contrast between the stimulation thresholds for the dorsal root entry zone and the dorsal column, allowing better focusing of the stimulus.
\end{abstract}

Keywords Spinal cord stimulation · intradural · simulation

\footnotetext{
Q. Huang and G. T. Gillies

Department of Mechanical Engineering, University of Virginia, Charlottesville, VA 22903, USA

H. Oya, O. E. Flouty, C. G. Reddy, and M. A. Howard Department of Neurosurgery, University of Iowa Hospitals and Clinics, Iowa City, IA 52242, USA

M. Utz

School of Chemistry, University of Southampton, Southampton SO17 1BJ, United Kingdom; E-mail: marcel.utz@ soton.ac.uk
}

\section{Introduction}

Spinal Cord Stimulation (SCS) was introduced to treat intractable pain in cancer patients by Shealy et al. in 1967 [33]. Since then, epidurally implanted stimulation devices have evolved significantly [2], and are now used to treat roughly 35,000 patients per year in the U.S. [19]. However, epidural SCS fails over the long term in up to half of all treated patients [6]. This is in part because the therapeutic window of stimulation strength between the onset of pain relief and the threshold for discomfort is very narrow, and tends to change with movement of the patient. This can be attributed to shunting effects by the high conductivity of cerebrospinal fluid (CSF) surrounding the spinal cord. With epidural electrodes, this results in current density leakage into neighbouring, not targeted structures of the spinal cord, producing paresthesia and other painful sensations due to activation of the dorsal root entry zones.

To overcome these limitations, we are developing a novel approach to SCS that places the electrode array directly on the pial surface of the spinal cord [16]. This intradural approach to spinal cord stimulation (ISCS) holds promise of being a more focused form of neuromodulation than epidural SCS (ESCS), thus potentially offering a wider therapeutic window and careful targeting of specific fibre bundles within the dorsal columns. Our initial goal is to develop this modality for the treatment of medically refractory pain, but future applications may include movement disorders associated with neurodegenerative diseases and spinal cord injury. While communication of stimulation signals into the intradural space raises technical problems, it may be possible to address these through judicious design of the implant and surgical tools $[16,17,26]$, as 
well as by using inductively coupled, wireless transmission protocols [34].

We have recently performed an extensive in vivo study of intradural SCS in a large animal model [7,8, 10]. Among other results, this work has shown that the threshold stimulation voltage for evoking responses in the somatosensory cortex is much lower when the stimulation electrodes are in direct contact with the pial surface.

The development and optimisation of a clinically useful intradural electrode array is a highly complex task. While animal studies provide invaluable highlevel information on somatosensory response and biocompatibility, they are costly, and they do not yield insight into the details of the electrophysiological response. Computational modelling is therefore indispensable in order to guide the design of electrode geometries and stimulation protocols.

The gate theory of pain proposed by Melzack and Wall [24] in 1965 still provides the framework for current understanding of the mechanisms behind pain management by spinal cord stimulation. It postulates a neurophysiological gate in the spinal cord. By stimulating large afferents within the dorsal column (DC), the transmission of noxious information (viz., pain signals) through the spinal cord to the brain can be attenuated, as demonstrated by Shealy et al. [33]. This has motivated a substantial body of work aimed at understanding neural modulation by external electrical stimuli [23, 28, 29, 41] based on electrophysiological models of signal transmission in myelinated and nonmyelinated axons $[4,9,13,23]$ in combination with numerical simulation of the electrical potential distribution in the spinal canal [3, 30, 31, 38]. Struijk, Holsheimer et al. developed a three-dimensional volumeconductor model of ESCS and used it to investigate how the stimulation of DC fibres threshold varied with several different factors, including the configuration of stimulating electrodes [38], and anatomic parameters of the nerve fibres $[36,37]$. The excitation of dorsal root (DR) fibres was studied systematically by Struijk, Holsheimer and Boom [35]. A significant amount of work has been dedicated to the impact of the position and configuration of the stimulating electrodes $[15,18,42]$. The results, showing the influence of electrode configuration, fibre geometry and thickness of the CSF layer on the effectiveness of ESCS, have been validated by clinical observations $[1,11,40]$. This computational approach evolved into the University of Twente Spinal Cord Stimulation Software (UT-SCS) package, which has now become the standard means of modelling the performance characteristics of advanced ESCS systems $[30,31]$.
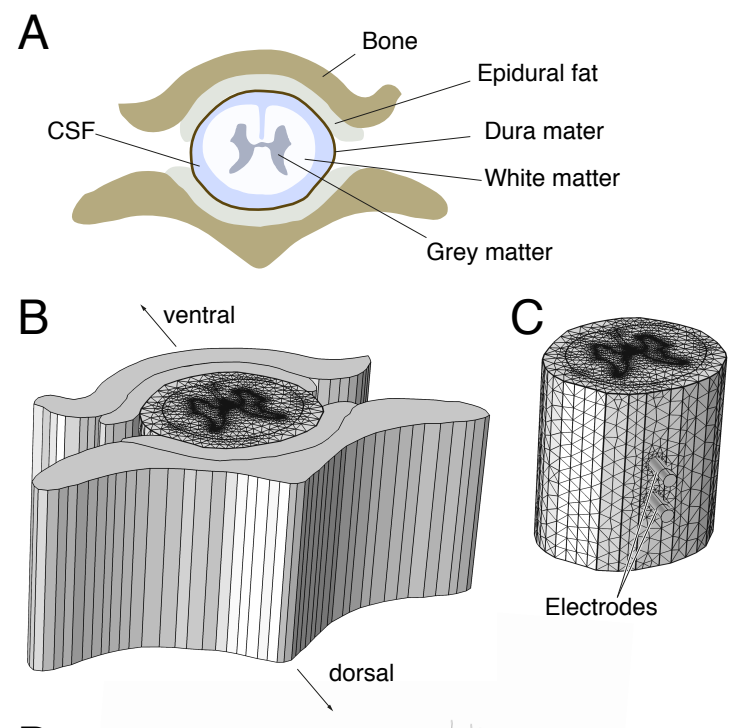

D

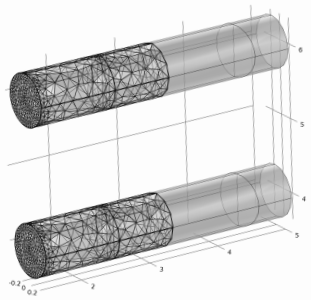

Fig. 1: A: Schematic cross section of the thoracic spinal canal. B: Computational model obtained from pyramidal extension of the cross section shown in A; only the spinal cord and the CSF layer are explicitly modelled, as indicated by the FEM mesh. C: Finite element computational domain with the surrounding tissue removed. The electrodes are represented as cylindrical objects. D: Detail of the electrode and tip and surface mesh.

In the present article, we use a similar approach to treat intra-dural (ISCS) electrode arrangements in order to test the hypothesis that intradural stimulation offers an advantage in efficiency and specificity over the traditional extradural arrangement.

\section{Method}

Our computational approach is based on a finite element simulation that treats the spinal canal tissues as an ohmic volume conductor $[3,5,38]$ in order to predict the electric potential distribution. The resulting potentials at the locations of nodes of Ranvier are then used as input to a simple nerve circuit model $[4,9,13,15,23,35-37]$. This provides estimates of the sub-threshold depolarisation voltages at the nodes of Ranvier, which are used to predict which axons are 

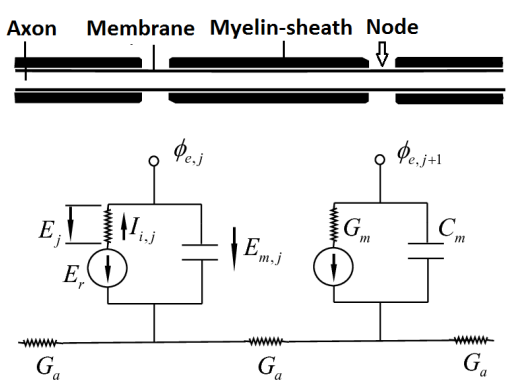

Fig. 2: The circuit model used for fibre segmentation [4]. Subscripts $e$ and $j$ represent the external potential $\phi_{e, j}=\phi_{e}\left(\mathbf{r}_{j}\right)$, where $\mathbf{r}_{j}$ is the location of fibre node $j$; $G_{a}$ and $G_{m}$ denote the nodal axoplasmic and membrane conductances, respectively, $E_{r}$ is the resting potential, $E_{m, j}$ the membrane potential, $C_{m}$ is the membrane capacitance, and $E_{j}$ denotes the depolarisation at node $j$.

likely to be recruited by a given positioning of the electrodes, and to estimate the necessary stimulus voltages or currents. The axonal excitation threshold was adopted from the nerve excitation standard for short excitation pulse given by Warman, Grill and Gurand [41] and the study of single nerve fibre excitation under time dependent conditions [43].

The COMSOL multiphysics package (versions 4.2a and 4.3a) was used to generate a 3-D finite element volume conductor representation of the anatomical structure of the spinal cord (Fig. 1) which incorporates the conductivities of the relevant tissues, as listed in Table 1.

The structure of the spinal cord varies slowly in the rostral-caudal direction. Since the relevant area for our purposes extends only over a few $\mathrm{cm}$ in length, the cross-section was taken as constant $[3,37,38]$. A pyramidal 3-D model was extruded from the transverse geometry of the spinal cord as determined by MR imaging (Fig. 1B). The length of the spinal cord segment was $20 \mathrm{~mm}$. Edge effects at the ends were minimised by applying periodic boundary conditions. The bipolar stimulation in the model was designed to match the experimental set-up, with an electrode pair positioned along the longitudinal direction of spinal cord. The electrodes (Fig. 1D) are $0.5 \mathrm{~mm}$ in diameter with a $1 \mathrm{~mm}$ gap between the edges. Simulations were run under conditions of constant prescribed current, with the anode and cathode of the bipolar stimulator set to the same current with positive and negative sign, respectively. Epidural and intradural stimulation were simulated by varying the position of the electrodes in the vental-dorsal direction, keeping all other parameters of the model constant.
The volume conductor model consists of four major domains: the CSF, the white matter, the grey matter and the electrodes. The finite element method was used to compute the potential distribution $\phi_{e}(\mathbf{r})$ as an approximate solution to the Laplace equation $\nabla^{2} \phi_{e}=0$. The current density $\mathbf{J}(\mathbf{r})$ is then obtained from the generalized version of Ohm's Law $\left(\mathbf{J}=\sigma \cdot \mathbf{E}, \mathbf{E}=-\nabla \phi_{e}\right)$. Here $\phi_{e}$ is the electrical potential and the subscript $e$ represents the external voltage outside the fibre nodes; $\mathbf{E}$ is the electric field; $\mathbf{J}$ is the current density, and $\sigma$ is the conductivity tensor.

While the conductivities (Table 1) of CSF and grey matter are isotropic, white matter is a better conductor in the axial than in the transverse direction. The electrodes are made from platinum with a conductivity of $9.40 \times 10^{6} \mathrm{~S} / \mathrm{m}$ [32]. Due to this very high value compared to all other components, we treat the electrodes as perfect conductors, and impose a current or voltage terminal boundary condition on their circular end surfaces. Conversely, both epidural fat and vertebral bone have only negligible electrical conductivities. Therefore, the outer surface of the dura is taken to be an insulator in the current model, and the computational domain includes only the CSF layer, front surface of the electrodes, and the spinal cord itself (Fig. 1C). Extradural stimulation is simulated by placing the conducting front surfaces of the electrodes outside of, but still in contact with the CSF. A 3-D free tetrahedral mesh was used, as shown in Fig. $1 \mathrm{~B}$ and $\mathrm{C}$. The mesh density on the conducting surface of the electrodes has been imposed by restricting the element size there to $<0.1 \mathrm{~mm}$. In the rest of the computational domain, the element size depends on the local geometry in the range between $0.2 \mathrm{~mm}$ and $1.6 \mathrm{~mm}$, with smaller sizes near sharp edges and boundaries. Convergence of the computed potential distributions to within $5 \%$ with respect to mesh density has been ensured by consecutive refinement of the mesh. The voltage distribution $\phi_{e}(\mathbf{r})$ in the spinal cord was exported on a Cartesian grid for use in the nerve fibre model with a vertical step size of $0.5 \mathrm{~mm}$ and transverse resolution of $0.1 \mathrm{~mm}$.

Table 1: Tissue conductivities used in the present model [25].

\begin{tabular}{cc}
\hline Material & Conductivity $(\mathrm{S} / \mathrm{m})$ \\
\hline Cerebrospinal fluid & 1.7 \\
White Matter (longitudinal) & 0.6 \\
White Matter (transverse) & 0.08 \\
Grey Matter & 0.23 \\
Platinum Electrodes & $9.43 \times 10^{6}$ \\
\hline
\end{tabular}




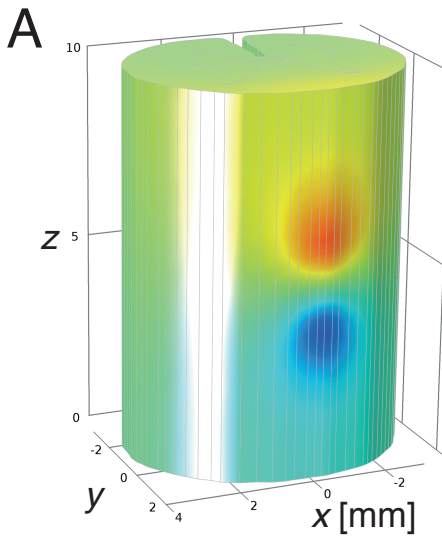

$\mathrm{B}$

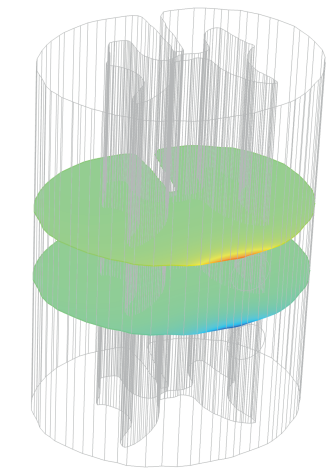

C

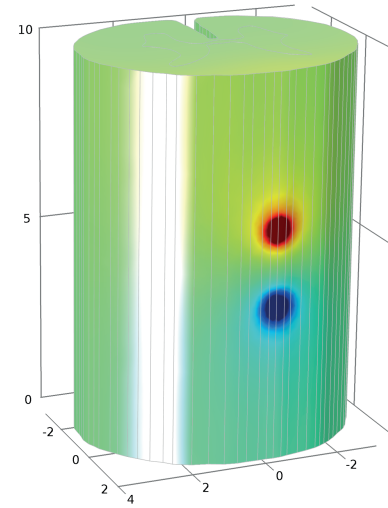

$\mathrm{D}$

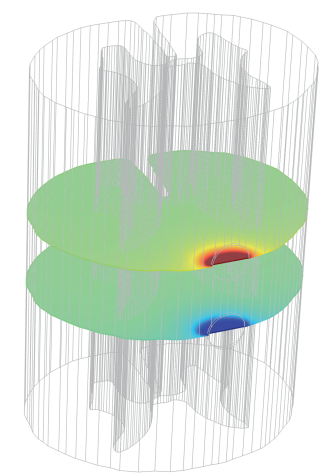

$\phi_{e}[\mathrm{~V}]$

0.25

0

$-0.25$

Fig. 3: Electrical potential caused by $1 \mathrm{~mA}$ stimulation current on the surface (A and $\mathrm{C}$ ) and over the cross-section of the spinal cord (B and D). In A and B, the electrodes placed at the surface of the CSF layer, whereas in C and $\mathrm{D}$ they are in direct contact with the pial surface.

We used the nerve fibre model of McNeal [23], which employs the electrical network shown in Fig. 2 to represent the axonal electrophysiology. This approach assumes that the radius of the nerve fibre is small enough so that the external voltage at any individual node is largely constant, and the presence of the nerve fibre does not influence the external electrical field.

The potential $\phi_{e, j}=\phi_{e}\left(\mathbf{r}_{j}\right)$ at node $j$ was used to calculate the internal depolarization voltage in a hypothetical axon running vertically through the system at that location. Since the goal of the present simulations was only to identify the spatial distribution of axons likely to be recruited by the a short-pulse stimulus, it was sufficient to use a sub-threshold description [23], in which the trans-membrane conductance is assumed to be constant. The recruitment area is then found by the region where the depolarisation reaches the threshold value of approximately $25 \mathrm{mV}$, corresponding to a pulse excitation duration of $20 \mu \mathrm{s}$ [41].

A fibre diameter of $5 \mu \mathrm{m}$ was assumed, with a spacing of the nodes of Ranvier of $500 \mu \mathrm{m}$. All other input parameters are based on the anatomical properties of the nerve fibre used by Coburn [4]. The membrane conductance $G_{m}$ is assumed to be independent of time. Obviously, in reality, this condition is violated when the threshold depolarisation is exceeded. However, even in this case, the membrane conductance changes on a time scale of milliseconds, much longer than the duration of the excitation pulses considered here $(20 \mu \mathrm{s})$ $[9,13]$. In order to estimate the recruitment area of an electrode arrangement, McNeal's model is therefore sufficient, and a full treatment of the non-linear axonal response $[9,13]$ is not necessary.

\section{Results}

Fig. 3 shows the potential $\phi_{e}(\mathbf{r})$ in the spinal cord resulting from $1 \mathrm{~mA}$ electrode current. Epidural stimulation, represented in the present model by placement of the electrodes just inside the surface of the CSF layer, leads to a diffuse potential distribution on the surface of the spinal cord (Fig. 3A). By contrast, placing the 

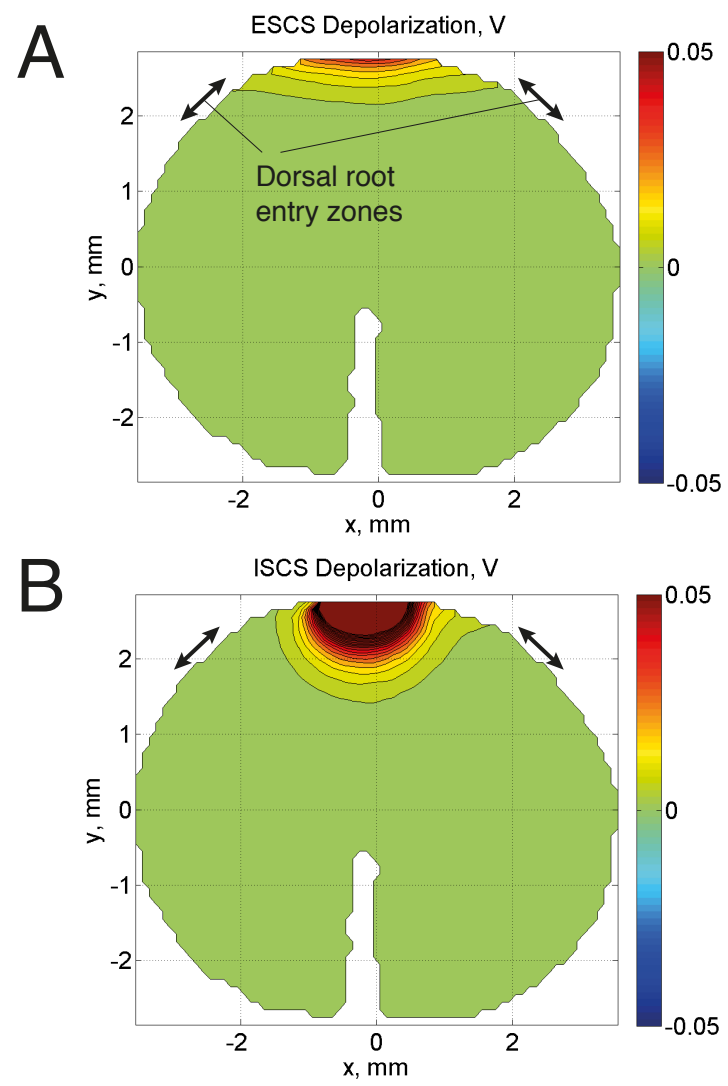

Fig. 4: The predicted depolarisation voltage in a "standard" axon running vertically through the spinal cord, plotted at the height of the cathode. A: ESCS with $10 \mathrm{~mA}$ stimulus current. B: ISCS with $1 \mathrm{~mA}$ stimulus current.

electrodes in direct contact with the spinal cord surface leads to a more defined potential distribution, where the area on the surface of the spinal cord that sees significant voltages is not much larger than the electrode surface (Fig. 3C).

These differences are even more manifest in the potential distribution inside the spinal cord. In the epidural arrangement, the profile extends over about $4 \mathrm{~mm}$ laterally, but is restricted to a strip within $200 \mu \mathrm{m}$ of the surface of the spinal cord (Fig. 3B). By contrast, the signal penetrates much more deeply when the electrodes are in contact with the pial surface (Fig. 3D). Fig. 4 shows cross section views of the depolarisation voltages for both both ESCS and ISCS. The cross sections are taken at the height of the cathode. The estimated recruitment area is given by the contour at $25 \mathrm{mV}$. In the case of ESCS, only a thin slice of axons in the dorsal column are excited by a $10 \mathrm{~mA}$ pulse. Increase of the excitation intensity spreads the recruitment area in depth and sideways, towards the dorsal

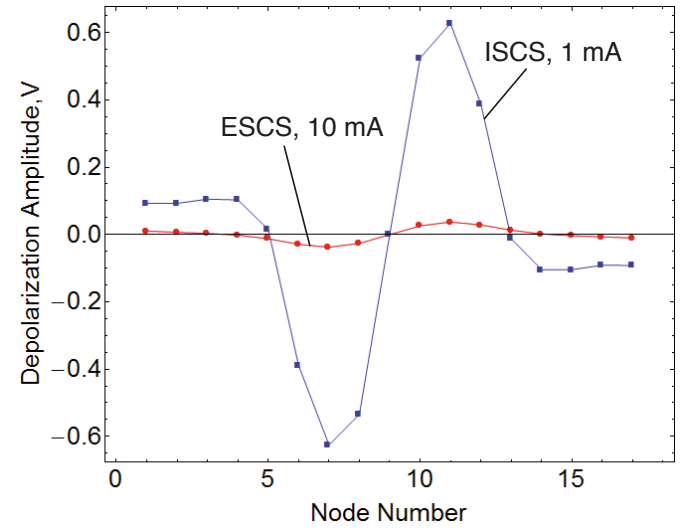

Fig. 5: The depolarization along a nerve fibre in the dorsal column for the case of ESCS and ISCS. Red: ESCS; Blue: ISCS.

root entry zone. By contrast, the recruitment area resulting from electrodes placed directly on the spinal cord surface is much more isotropically shaped, with a lateral extension comparable to the penetration depth.

The resulting depolarisation voltages along the nodes of Ranvier in a hypothetical axon running vertically through the dorsal column $250 \mu \mathrm{m}$ below the surface are shown in Fig. 5. The nodes on the left hand side of the plot, corresponding to the anode side of the electrode assembly, are hyper-polarised, whereas the ones on the right, which are closer to the the cathode, are depolarised. While the depolarisation barely reaches $40 \mathrm{mV}$ at an electrode current of $10 \mathrm{~mA}$ in an epidural arrangement, more than $600 \mathrm{mV}$ are obtained with the subdural electrode position, even the electrode current is ten times smaller ( $1 \mathrm{~mA})$.

\section{Discussion}

The shape of the ESCS recruitment area (Fig. 4A) agrees well with the results reported by several other authors $[12,14,21,37]$. Only a shallow slice on the dorsal surface of the spinal cord is excited. While deeper recruitment can be achieved by stronger stimuli, this will also affect the dorsal root entry zones, which causes undesirable side effects. We suspect this to be the cause for the narrow therapeutic window associated with ESCS. By contrast, as is evident from the shape of the potential distribution (Fig. 3D) and the depolarisation voltages (Fig. 4B), the intradural stimulation profile is much more sharply defined. Since this makes it easier to avoid undesired stimulation of the dorsal root entry zone, it is reasonable to expect that a wider therapeutic range of stimulation conditions can be obtained from intradural electrode arrangement. From the shape 
of the affected area shown in Fig. 4B, it is obvious that the axonal recruitment can be restricted to a thin layer beneath the electrode in ISCS, if the stimulation current is sufficiently small. Most significantly, the lateral extents of the recruitment area will quite precisely reflect the dimensions of the electrodes, with little sideways spillage, thus avoiding undesirable activation of the dorsal root entry zone.

The voltages produced in the spinal tissue by a stimulation current of $1 \mathrm{~mA}$ are in the vicinity of $250 \mathrm{mV}$ in the intradural case, but reach only $100 \mathrm{mV}$ with extradural electrodes. Since the axon activation thresholds are around $25 \mathrm{mV}$, a much smaller stimulation current (of the order of $100 \mu \mathrm{A}$ or less) would be sufficient in the case of ISCS. This greater sensitivity to current of the ISCS arrangement corresponds to a significant increase in electrode impedance, which in our simulations grew from $650 \Omega$ in the extradural arrangement to $1200 \Omega$ when the electrodes were in contact with the spinal cord. This means that similar stimulation can be achieved at roughly 50 times smaller power deposition in the tissue, thus enabling the use of novel high-frequency stimulation protocols, which would be impractical in ESCS due to power supply limitations and possible tissue heating.

In summary, the simulation results suggest that ISCS (a) requires much smaller stimulus currents and voltages, (b) provides a much more focused recruitment area, and (c) may offer a wider therapeutic window of stimulation.

It should be noted that the results depend directly on the values of the tissue conductivities, which are subject to considerable uncertainty and to variations from one individual to another. However, the main features of the potential distributions are defined by just two aspects: the high conductivity of the CSF, and the anisotropy in the conductivity in white matter. While we expect variations in the absolute conductivities of up to $10 \%$ between individuals, these features are general, and we therefore expect the conclusions to be robust.

The results presented here are consistent with those from studies on an ovine model that have recently been conducted by our group $[7,8,10]$. In those experiments, the response of the somatosensory cortex was recorded in anesthesized sheep during stimulation with subdural and epidural spinal cord electrodes. A bipolar neurostimulator was used with dimensions very similar to the one studied here. As predicted by the simulation results, the necessary stimulation voltages were much lower when the electrodes were in direct contact with the pial surface: It was found that a response on the anterior-most bank of the supra-sylvain sulcus could be evoked reliably with only $1 \mathrm{~V}$ stimulation voltage if it was applied inside the dura, whereas $5 \mathrm{~V}$ were necessary for epidural stimulation [8].

In the same vein, the threshold stimulation voltage for a somatosensory response was measured as a function of the distance between the electrodes and the pial surface. These latter experiments were conducted with the dura open, and the electrodes surrounded by CSF. The threshold stimulation voltage was $0.2 \mathrm{~V}$ when the electrode was in direct contact with the spinal cord, and increased gradually to $1 \mathrm{~V}$ at $3 \mathrm{~mm}$ separation. In addition, the stimulation threshold depended strongly on the lateral placement of the electrode when it was in direct contact with the pial surface, whereas this specificity was lost at $3 \mathrm{~mm}$ separation. This corresponds to the finding from the present study that highly specific stimulation is only possible with direct contact between the electrodes and the pial surface.

The improved distribution of current densities offered by ISCS will loosen constraints on device designs, such as those now governing the electrode configurations used in ESCS. A recent example of the great care that must be taken in the latter is given in [12], which evaluates the electric field and current density distributions associated with several types of epidural electrode placements and geometries. A similar FEM assessment for penetrating electrodes is also available [22]. Moreover, with improved current density distributions, it may be possible to augment the progress made to date in the use of SCS to treat patients with spinal cord injury. Ladenbauer et al. [20] have carried out finite element modelling of the stimulation process for this purpose, evaluating epidural and surface-electrode approaches to such therapies. Our ISCS approach expands this range of potential treatment options. Ultimately, to maximise their predictive value, FEM models incorporating responses of the spinal cord tissues to both mechanical forces and electrical stimuli will be needed. This would make it possible to take into account a number of physiologically important phenomena, for instance, how the kinematics of axon couplings [27] might influence the electrical impedances of the fibre clusters.

While the computational approach presented here will provide important guidance in the design of intradural electrode arrays, its predictive capabilities are limited by the simple nature of the electrical tissue properties employed. A more realistic treatment would take into account reactive components of the tissue response [39]. This would allow more complete simulation of the time-dependent current and potential distributions following a stimulus pulse, and would enable the simultaneous computational optimisation of stim- 
ulus timing and electrode geometry. A particular challenge in this context is the estimation of realistic values for the tissue capacitance, and the calibration of the computational model to measured impedances. Efforts towards such computations are currently underway in our group, and will be reported on at a later occasion.

\section{Conclusions}

A simple finite element model of intra- and extra-dural spinal cord stimulation has been developed. The results confirm recent experimental observations that intradural stimulation requires much smaller stimulus inputs, and predict the magnitude of the required stimulation voltages semi-quantitatively. The model also predicts that the stimulus can be focused on the dorsal column much more accurately with intradural arrangements.

\section{Acknowledgment}

The authors thank Prof. J. Buitenweg of U. Twente for helpful discussions, and their University of Iowa colleagues H. Chen and H. Kawasaki for technical assistance with the experimental part of the project. Partial support by the University of Virginia Biomedical Innovation Fund is gratefully acknowledged.

\section{References}

1. Alo, K.M., Holsheimer, J.: New trends in neuromodulation for the management of neuropathic pain. Neurosurgery 50(4), 690-704 (2002)

2. Atkinson, L., Sundaraj, S., Brooker, C., OCallaghan, J., Teddy, P., Salmon, J., Semple, T., Majedi, P.: Recommendations for patient selection in spinal cord stimulation. Journal of Clinical Neuroscience 18(10), 1295-1302 (2011)

3. Coburn, B.: Electrical stimulation of the spinal cord: twodimensional finite element analysis with particular reference to epidural electrodes. Medical and Biological Engineering and Computing 18(5), 573-584 (1980)

4. Coburn, B.: A theoretical study of epidural electrical stimulation of the spinal cord-part ii: Effects on long myelinated fibers. Biomedical Engineering, IEEE Transactions on (11), 978-986 (1985)

5. Coburn, B., Sin, W.K.: A theoretical study of epidural electrical stimulation of the spinal cord part i: Finite element analysis of stimulus fields. Biomedical Engineering, IEEE Transactions on (11), 971-977 (1985)

6. Eldabe, S., Kumar, K., Buchser, E., Taylor, R.S.: An analysis of the components of pain, function, and health-related quality of life in patients with failed back surgery syndrome treated with spinal cord stimulation or conventional medical management. Neuromodulation: Technology at the Neural Interface 13(3), 201-209 (2010)

7. Flouty, O., Oya, H., Kawasaki, H., Wilson, S., Reddy, C.G., Jeffery, N.D., Brennan, T.J., Gibson-Corley, K.N., Utz, M., Gillies, G.T., et al.: A new device concept for directly modulating spinal cord pathways: initial in vivo experimental results. Physiological measurement 33(12), 2003 (2012)
8. Flouty, O.E., Oya, H., Kawasaki, H., Reddy, C.G., Fredericks, D.C., Gibson-Corley, K.N., Jeffery, N.D., Gillies, G.T., Howard III, M.A.: Intracranial somatosensory responses with direct spinal cord stimulation in anesthetized sheep. PloS one 8(2), e56,266 (2013)

9. Frankenhaeuser, B., Huxley, A.: The action potential in the myelinated nerve fibre of xenopus laevis as computed on the basis of voltage clamp data. The Journal of Physiology 171(2), 302-315 (1964)

10. Gibson-Corley, K.N., Oya, H., Flouty, O., Fredericks, D.C., Jeffery, N.D., Gillies, G.T., Howard III, M.A.: Ovine tests of a novel spinal cord neuromodulator and dentate ligament fixation method. Journal of Investigative Surgery 25(6), 366-374 (2012)

11. He, J., Barolat, G., Holsheimer, J., Struijk, J.J.: Perception threshold and electrode position for spinal cord stimulation. Pain 59(1), 55-63 (1994)

12. Hernández-Labrado, G.R., Polo, J.L., López-Dolado, E., Collazos-Castro, J.E.: Spinal cord direct current stimulation: finite element analysis of the electric field and current density. Med Biol Eng Comput 49(4), 417-429 (2011)

13. Hodgkin, A.L., Huxley, A.F.: A quantitative description of membrane current and its application to conduction and excitation in nerve. The Journal of physiology 117(4), 500 (1952)

14. Holsheimer, J.: Which Neuronal Elements are Activated Directly by Spinal Cord Stimulation. Neuromodulation: Technology at the Neural Interface 5(1), 25-31 (2002)

15. Holsheimer, J., Struijk, J.J.: Electrode geometry and preferential stimulation of spinal nerve fibers having different orientations: a modeling study. In: Engineering in Medicine and Biology Society, 1992 14th Annual International Conference of the IEEE, vol. 4, pp. 1374-1375. IEEE (1992)

16. Howard, M., Utz, M., Brennan, T., Dalm, B., Viljoen, S., Jeffery, N., Gillies, G.: Intradural approach to selective stimulation in the spinal cord for treatment of intractable pain: design principles and wireless protocol. Journal of Applied Physics 110(4), 044,702-044,702 (2011)

17. Howard, M., Utz, M., Brennan, T., Dalm, B., Viljoen, S., Kanwal, J., Gillies, G.: Biophysical attributes of an in vitro spinal cord surrogate for use in developing an intradural neuromodulation system. Journal of Applied Physics 110(7), 074,701-074,701 (2011)

18. Koole, P., Holsheimer, J., Struijk, J.J., Verloop, A.J.: Recruitment characteristics of nerve fascicles stimulated by a multigroove electrode. Rehabilitation Engineering, IEEE Transactions on 5(1), 40-50 (1997)

19. Kumar, K., Bishop, S.: Financial impact of spinal cord stimulation on the healthcare budget: a comparative analysis of costs in canada and the united states: Clinical article. Journal of Neurosurgery: Spine 10(6), 564-573 (2009)

20. Ladenbauer, J., Minassian, K., Hofstoetter, U.S., Dimitrijevic, M.R., Rattay, F.: Stimulation of the Human Lumbar Spinal Cord With Implanted and Surface Electrodes: A Computer Simulation Study. Ieee T Neur Sys Reh 18(6), 637-645 (2010)

21. Manola, L., Holsheimer, J., Veltink, P.: Technical Performance of Percutaneous Leads for Spinal Cord Stimulation: A Modeling Study. Neuromodulation: Technology at the Neural Interface 8(2), 88-99 (2005)

22. McIntyre, C.C., Grill, W.M.: Finite Element Analysis of the Current-Density and Electric Field Generated by Metal Microelectrodes. Annals of Biomedical Engineering 29(3), 227-235 (2001)

23. McNeal, D.R.: Analysis of a model for excitation of myelinated nerve. Biomedical Engineering, IEEE Transactions on (4), 329-337 (1976) 
24. Melzack, R., Wall, I.: Pain mechanisms: a new theory. Science 50, 971-979 (1965)

25. Oakley, J.C., Prager, J.P.: Spinal cord stimulation: mechanisms of action. Spine 27(22), 2574-2583 (2002)

26. Oya, H., Reddy, C., Dahdaleh, N., Wilson, S., Howard III, M., Jeffery, N., Utz, M., Gillies, G.: Applier tool for intradural spinal cord implants. Journal of medical engineering \& technology 36(3), 169-173 (2012)

27. Pan, Y., Shreiber, D.I., Pelegri, A.A.: A Transition Model for Finite Element Simulation of Kinematics of Central Nervous System White Matter. IEEE Transactions on Biomedical Engineering 58(12), 3443-3446 (2011)

28. Rattay, F.: Analysis of models for external stimulation of axons. Biomedical Engineering, IEEE Transactions on (10), 974-977 (1986)

29. Rattay, F.: Analysis of models for extracellular fiber stimulation. Biomedical Engineering, IEEE Transactions on 36(7), 676-682 (1989)

30. Sankarasubramanian, V., Buitenweg, J., Holsheimer, J., Veltink, P.: Electrode alignment of transverse tripoles using a percutaneous triple-lead approach in spinal cord stimulation. Journal of Neural Engineering 8(1), 016,010 (2011)

31. Sankarasubramanian, V., Buitenweg, J.R., Holsheimer, J., Veltink, P.: Triple leads programmed to perform as longitudinal guarded cathodes in spinal cord stimulation: A modeling study. Neuromodulation: Technology at the Neural Interface 14(5), 401-411 (2011)

32. Shackelford, J.F., Alexander, W. (eds.): CRC Materials Science and Engineering Handbook, 3rd edn. CRC Press, Boca Raton (2001)

33. Shealy, C.N., Mortimer, J., Reswick, J.: Electrical inhibition of pain by stimulation of the dorsal columns. Anesth Analg 46, 489-491 (1967)

34. Song, S.H., Gillies, G.T., Howard III, M.A., Kuhnley, B., Utz, M.: Power and signal transmission protocol for a contactless subdural spinal cord stimulation device. Biomedical microdevices 15(1), 27-36 (2013)

35. Struijk, J.J., Holsheimer, J., Boom, H.B.: Excitation of dorsal root fibers in spinal cord stimulation: a theoretical study. IEEE transactions on biomedical engineering 40(7), 632639 (1993)

36. Struijk, J.J., Holsheimer, J., van der Heide, G.G., Boom, H.B.: Recruitment of dorsal column fibers in spinal cord stimulation: influence of collateral branching. Biomedical Engineering, IEEE Transactions on 39(9), 903-912 (1992)

37. Struijk, J.J., Holsheimer, J., Van Veen, B., Boom, H.B.: Epidural spinal cord stimulation: calculation of field potentials with special reference to dorsal column nerve fibers. Biomedical Engineering, IEEE Transactions on 38(1), 104110 (1991)

38. Struijk, J.J., Holsheimer, J., van Veen, B.K.: Analysis of dorsal column stimulation. In: Engineering in Medicine and Biology Society, 1988. Proceedings of the Annual International Conference of the IEEE, pp. 1692-1693. IEEE (1988)

39. Tracey, B., Williams, M.: Computationally efficient bioelectric field modeling and effects of frequency-dependent tissue capacitance. J Neural Eng 8(3), 036,017 (2011)

40. de Vos, C.C., Hilgerink, M.P., Buschman, H.P., Holsheimer, $\mathrm{J}$.: Electrode contact configuration and energy consumption in spinal cord stimulation. Neurosurgery $\mathbf{6 5}(6), 210-217$ (2009)

41. Warman, E.N., Grill, W.M., Durand, D.: Modeling the effects of electric fields on nerve fibers: determination of excitation thresholds. Biomedical Engineering, IEEE Transactions on 39(12), 1244-1254 (1992)

42. Wesselink, W., Holsheimer, J.: Electrical safety in spinal cord stimulation: current density analysis by computer modeling. In: Engineering in Medicine and Biology Society,
1995., IEEE 17th Annual Conference, vol. 2, pp. 11351136. IEEE (1995)

43. Woo, J., Miller, C.A., Abbas, P.J.: The dependence of auditory nerve rate adaptation on electric stimulus parameters, electrode position, and fiber diameter: a computer model study. Journal of the Association for Research in Otolaryngology 11(2), 283-296 (2010) 